\title{
Prevenção do Nascimento Prematuro: Importância da Monitorização das Contrações Uterinas
}

\author{
Prevention of Preterm Birth: Role of Uterine Contraction Monitoring \\ Eduardo Sérgio Borges da Fonseca, Roberto Eduardo Bittar, Marcelo Zugaib
}

\begin{abstract}
RESUMO
Objetivo: avaliar a relação entre alterações das contrações uterinas e parto prematuro. Metodologia: entre fevereiro de 1996 e junho de 1998, 73 gestantes com risco para o parto prematuro foram submetidas à monitorização externa das contrações uterinas, da $24^{a}$ à $34^{a}$ semana de gestação, duas vezes por semana, durante 60 minutos. O teste foi considerado positivo quando as contrações uterinas apresentavam freqüência maior ou igual a 4 contrações/hora antes da $30^{a}$ semana e a 6 contrações/hora após esta data.

Resultado: foram excluídas da análise 17 pacientes (23,28\%) por apresentarem patologias obstétricas ou evolução desfavorável para os resultados finais. Das 56 gestantes restantes, a incidência de partos prematuros espontâneos foi de 23,21\% (13/56). A freqüência média das contrações uterinas foi significativamente maior no grupo que evoluiu para o parto prematuro. O método revelou uma sensibilidade de 69,23\%, uma especificidade de 86,04\% e valores preditivos positivo e negativo de $60 \%$ e 90,24\%, respectivamente.

Conclusão: o teste negativo está associado a baixo risco de nascimento prematuro. Contudo, diante do teste positivo, torna-se necessária a associação com outros marcadores do parto prematuro para melhor identificar pacientes com risco elevado.
\end{abstract}

PALAVRAS-CHAVE: Contração uterina. Prematuridade. Trabalho de parto.

\section{Introdução}

Atualmente, a redução da prematuridade é um dos principais objetivos da Obstetrícia. O nascimento prematuro está associado com $75 \%$ da mortalidade neonatal e representa a causa mais freqüente de morbidade neonatal ${ }^{1}$.

Apesar dos avanços da Obstetrícia, a incidência da prematuridade permanece estável, em virtude da etiologia multifatorial, das dificuldades em se diagnosticar o trabalho de parto prematuro (TPP) e pelas controvérsias quanto à eficácia da tocólise ${ }^{2}$. Nos EUA, observou-se um aumento na última década, sendo que nos últimos três anos permanece constante em torno de $11 \%{ }^{3}$.

No Hospital das Clínicas da FMUSP, em

Clínica Obstétrica da Faculdade de Medicina da Universidade de São Paulo - FMUSP

Correspondência:

Eduardo Sérgio Borges da Fonseca

Av 11 de Junho, 1006/133

04041-003 - São Paulo - SP

e-mail:riedu@uol.com.br virtude do baixo nível sócio-econômico da população atendida e da elevada prevalência de gestações de alto risco, a média de nascimentos prematuros nos últimos anos foi de $22 \%$. A metade destes casos foi devida a partos prematuros espontâneos e o restante, decorrente de partos prematuros eletivos, conseqüentes a intercorrências clínicas e/ou obstétricas que impunham riscos fetais e/ou maternos ${ }^{4}$.

Uma alternativa para solucionar, em parte, algumas destas dificuldades seria a utilização de métodos de rastreamento para identificar uma população mais suscetivel ao nascimento antes da $37^{\text {a }}$ semana de gestação o que permitiria medidas mais precoces ${ }^{5}$.

Na década passada, Papiernik ${ }^{6}$ afirmou que a predição do parto prematuro poderia ser conseguida por meio da identificação de alguns fatores de risco demográficos e obstétricos, tais como: idade materna menor que 21 ou maior que 36 anos e baixo nível sócio-econômico, antecedente de parto pré-termo e estatura materna inferior a 1,52 m. Outros fatores de risco 
aventados foram gestação gemelar, sangramento vaginal no $2^{\circ}$ trimestre, amadurecimento cervical e aumento da atividade uterina antes da 29a semana de gestação. Entretanto, outros investigadores utilizando sistemas de pontuação de risco baseados nestes fatores obtiveram baixos valores preditivos ${ }^{7,8}$.

A exemplo de outros marcadores de risco, tais como a fibronectina fetal (fFN) $)^{9,10}$ e as alterações cervicais identificadas por meio do toque vaginal e da ultra-sonografia transvaginal $^{11,12,13}$; a monitorização das contrações uterinas têm sido igualmente empregada atualmente $^{14}$, pois trata-se de um marcador clínico possivel de ser utilizado mesmo em locais desprovidos de grandes avanços tecnológicos.

Sua importância como um método preditivo para o parto prematuro baseia-se em estudos realizados na década de 50 , segundo os quais o útero exibe contrações rítmicas e arrítmicas ao longo da gestação, que aumentam em freqüência e duração pelo menos 2 a 3 semanas antes do parto $^{15,16}$.

É consenso que as gestantes que evoluem para o parto prematuro apresentam uma maior freqüência de contrações uterinas ${ }^{15,16,17}$. Alguns estudos têm demonstrado uma relação positiva entre a presença de 4 contrações/h ou mais antes da $30^{a}$ semana de gestação e de 6 ou mais após esta data, e o nascimento antes da 37 semana. De forma inversa, as que não apresentam parto prematuro possuem freqüência máxima menor ou igual a 3 contrações/h antes da $28^{a}$ semana de gestação e menor ou igual a 5 contrações/h entre a $29^{a}$ e $32^{\text {a } 18}$.

Os estudos comparativos entre a monitorização externa das contrações uterinas com o tocodinamômetro e a autopalpação demonstram melhores resultados quando utilizado o primeiro método ${ }^{16,18}$. Em tais estudos o diagnóstico do TPP se deu em 92\% dos casos monitorizados com dilatação cervical inferior a 2 $\mathrm{cm}$, o que permitiu um ganho de tempo intra-útero significativamente maior nesta população.

A capacidade de predição do parto prematuro por meio deste método foi avaliada por vários autores que realizaram estudos prospectivos, porém os resultados são controversos, em virtude das diferenças metodológicas existentes, tais como a época e a duração do exame, o número de contrações aceitáveis como normal e o padrão ideal adotado. Contudo, a maioria dos estudos tem considerado a freqüência normal máxima menor ou igual a 3 contrações $/ h^{16,18,19,20,21}$. Main et al. ${ }^{16}$ consideram a freqüência superior ou igual a 4 contrações/h, com 35 segundos ou mais e amplitude maior ou igual a $5 \mathrm{~mm}$, preditivas do parto pré-termo. Apesar de estes estudos terem revelado sensibilidade variando entre 57 e 85,7\%, sua interpretação é prejudicada pelo fato de várias pacientes terem sido submetidas à tocólise.

Portanto, como ainda não há consenso sobre a real eficácia da monitorização das contrações uterinas por meio da tocografia externa em gestantes com risco para o parto prematuro, tal método é motivo de controvérsia, sendo necessárias pesquisas adicionais a fim de demonstrar o seu real valor.

\section{Pacientes e Métodos}

No período de fevereiro de 1996 a junho de 1998, 73 gestantes de alto risco para o parto prematuro, entre a $24^{\mathrm{a}}$ e $34^{\mathrm{a}}$ semana de gestação, foram acompanhadas prospectivamente, sendo submetidas, duas vezes por semana, a monitorização das contrações uterinas durante 60 minutos por meio do monitor de cardiotocografia fetal da marca Hewlett-Packard - modelo M1351.

Dezessete $(23,28 \%)$ pacientes foram excluídas do estudo por apresentarem as seguintes intercorrências: doença hipertensiva específica da gestação (6), rotura prematura das membranas ovulares (3), diabetes gestacional (1), tocólise com evolução para o parto a termo (4) e abandono do pré-natal (3).

As consultas pré-natais foram quinzenais até a $28^{\mathrm{a}}$ semana e, após, semanais. Durante a primeira consulta as pacientes recebiam orientação a respeito dos sintomas de TPP e dos objetivos do estudo. As que optassem por participar assinavam um termo de consentimento aprovado pela Comissão de Ética para análise de projetos de pesquisa do HC-FMUSP (protocolo 256). Em nenhum dos casos foi instituída terapia profilática para o parto prematuro.

A idade gestacional foi calculada a partir da data da última menstruação (DUM) em concordância com uma ultra-sonografia realizada até a $12^{\text {a }}$ semana, ou por meio de dois exames ultra-sonográficos entre a $12^{\mathrm{a}}$ e $20^{\mathrm{a}}$ semana de gestação concordantes. Considerou-se parto prematuro aquele que ocorreu antes de 37 semanas completas de gestação (<259 dias).

O TPP foi definido pelos critérios de Herron et al. ${ }^{5}:$ 1) idade gestacional inferior a 37 semanas completas; 2) contrações uterinas a cada $5 \mathrm{~min}$ ou menos; 3) dilatação cervical de pelo menos 2 $\mathrm{cm}$ e 4) esvaecimento cervical de $80 \%$. Diante do mesmo, empregou-se o tratamento mais adequado para cada caso, dependendo do estágio clínico do trabalho de parto, segundo protocolo de normas e 
condutas específico.

Dentre as 56 gestantes restantes foram identificados os seguintes fatores de riscos: antecedente de parto prematuro espontâneo (37/ 56), miomas uterinos $(14 / 56)$, insuficiência cervical $(7 / 56)$ e malformações uterinas $(2 / 56)$.

A monitorização das contrações uterinas foi considerada positiva quando a freqüência das contrações era $>4$ contrações $/ \mathrm{h}$, antes da $30^{\underline{a}}$ semana de gestação, e $>6$ contrações/h após esta idade gestacional, com duração superior a 40 segundos. Estes valores foram obtidos por meio de uma revisão retrospectiva de vários estudos com o objetivo de maximizar a habilidade de rastreamento do teste ${ }^{16,17,19}$.

A análise estatística foi realizada utilizando o software EPI-INFO versão 6.04, fornecido pelo CDC ("Centers for Disease Control") de AtlantaEUA. Foram calculados os valores de freqüência de cada uma das variáveis maternas e neonatais. Os resultados foram expressos em média \pm desviopadrão (DP). Comparou-se o grupo cujo nascimento foi prematuro com o que evoluiu para o parto a termo, pelo teste de hipótese, teste de $\chi^{2}$ e teste exato de Fisher, quando aplicável, observando-se um nível de significância de 5\% e um intervalo de confiança de $95 \%$.

Foram calculadas, ainda, a sensibilidade, a especificidade, os valores preditivos (positivo e negativo) e o risco relativo do parto prematuro quando o teste de monitorização das contrações uterinas era positivo, tendo como padrão-ouro o nascimento prematuro.

\section{Resultados}

Dezessete mulheres (30,35\%) desenvolveram TPP e 13 destas evoluíram para o parto antes da 37a semana de gestação. Além disso, 4 gestantes responderam à inibição e foram excluídas do estudo. O nascimento prematuro ocorreu entre a $32^{\mathrm{a}}$ e $36^{\mathrm{a}}$ semana, sendo a média da idade gestacional neste grupo de 34 semanas $( \pm 1,6)$.

Não houve diferenças significativas quanto à idade, cor e estado civil entre o grupo com parto prematuro e a termo (Tabela 1).

Foram realizados em média $11( \pm 2,7)$ exames por paciente (variação de 6 a 18), perfazendo um tempo total de 660 minutos de registros tocográficos para cada gestante. A idade gestacional em que o primeiro exame foi realizado ocorreu na $24^{\mathrm{a}}( \pm 0,6)$ semana para o grupo prematuro e na $26^{\mathrm{a}}( \pm 0,6)$ semana para o grupo com parto a termo, não havendo, portanto, diferença estatisticamente significativa.
Tabela 1 - Características obstétricas e demográficas da população estudada.

\begin{tabular}{|c|c|c|c|}
\hline & Total & Pré-termo & Termo \\
\hline $\mathrm{n}$ & 56 & 13 & 43 \\
\hline Idade (anos) & $7,6( \pm 6,3)$ & $26,6( \pm 6,3)$ & $28,8( \pm 6,3)$ \\
\hline \multicolumn{4}{|l|}{ Cor } \\
\hline Branca & $62,5 \%$ & $69,2 \%$ & $60,5 \%$ \\
\hline Não-Branca & $37,5 \%$ & $30,8 \%$ & $39,5 \%$ \\
\hline \multicolumn{4}{|l|}{ Paridade } \\
\hline Nulípara & $21,1 \%$ & $7,7 \%$ & $25,6 \%$ \\
\hline Primípara & $37,5 \%$ & $30,8 \%$ & $39,5 \%$ \\
\hline Multípara & $41,1 \%$ & $61,5 \%$ & $34,9 \%$ \\
\hline \multicolumn{4}{|l|}{ Fatores de risco } \\
\hline PPA & $66,7 \%$ & $100 \%$ & $55,8 \%$ \\
\hline Mioma & $25,0 \%$ & $7,7 \%$ & $30,2 \%$ \\
\hline IC & $12,5 \%$ & $0 \%$ & $16,3 \%$ \\
\hline MFU & $3,57 \%$ & $0 \%$ & $4,6 \%$ \\
\hline IG no parto (semana) & $38( \pm 1,4)$ & $34( \pm 1,6)$ & $39( \pm 1,6)$ \\
\hline \multicolumn{4}{|c|}{$\begin{array}{l}\text { PPA (parto prematuro anterior) } \\
\text { IC (incompetência cervical) } \\
\text { MFU (malformação uterina) } \\
\text { IG (idade gestacional) }\end{array}$} \\
\hline
\end{tabular}

A análise da freqüência média global de contrações uterinas revelou a presença de 3,5 contrações $/ \mathrm{h}$, sendo que o grupo prematuro apresentou uma média de 8 contrações/h contra apenas 2 contrações/h do grupo com parto a termo ( $\mathrm{p}<0,05)$. Como demonstrado na Tabela 2 , a média da freqüência de contrações uterinas por idade gestacional foi significativamente maior entre as gestantes com parto prematuro.

Tabela 2 - Relação entre a freqüência das contrações uterinas e o nascimento a termo e prematuro.

\begin{tabular}{llrrrr}
\hline Contrações/hora & \multicolumn{2}{c}{ Prematuros } & \multicolumn{2}{c}{ Termo } & Total \\
\hline & $\mathrm{n}$ & \multicolumn{1}{c}{$\%$} & \multicolumn{1}{c}{$\mathrm{n}$} & $\%$ & \\
$<2$ & 1 & 4,0 & 24 & 96 & 25 \\
3 a 4 & 2 & 33,4 & 4 & 66,6 & 6 \\
5 a 7 & 3 & 27,3 & 8 & 72,7 & 11 \\
8 a 10 & 2 & 40,0 & 3 & 60,0 & 5 \\
$>10$ & 5 & 55,5 & 4 & 44,5 & 9 \\
\hline
\end{tabular}

Ao considerarmos o número máximo de contrações por hora entre as pacientes, observamos uma relação significativa entre as contrações e o parto prematuro. Como demonstra a Figura 1, quanto maior o número de contrações maior a freqüência de partos prematuros e quanto menor o número de contrações maior a freqüência de partos a termo. 


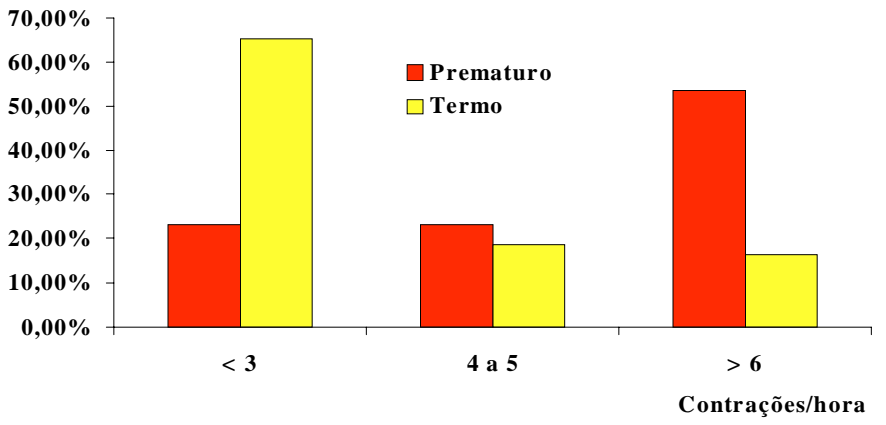

Figura 1 - Freqüência de nascimento a termo e prematuro em relação às contrações.

Das 25 pacientes com contração máxima menor ou igual a $2 / \mathrm{h}$, apenas uma (4\%) teve parto prematuro e, entre as seis gestantes com freqüência máxima de 3 contrações/h, observamos dois $(33,4 \%)$ partos prematuros. Deste modo, a incidência de prematuros nestas 31 gestantes $(9,7 \%)$ foi menor que no grupo total $(23,21 \%)$. Três entre 11 mulheres $(27,3 \%)$ com freqüência máxima entre 5 a 7 contrações/h apresentaram parto prematuro. Entretanto, quando a monitorização das contrações uterinas registrou uma freqüência máxima maior ou igual a 8 contrações $/ \mathrm{h}$, a prevalência de nascimento prematuro (7/14 - 50\%) foi, praticamente, o dobro da população geral (Tabela 2).

Como demonstrado na Figura 2, 41 gestantes apresentaram testes negativos $(73,21 \%)$ e 15 testes positivos (26,79\%). Das pacientes com teste negativo, apenas quatro $(9,75 \%)$ evoluíram com parto pré-termo. Dentre as com teste positivo, ocorreram nove partos prematuros (60\%).

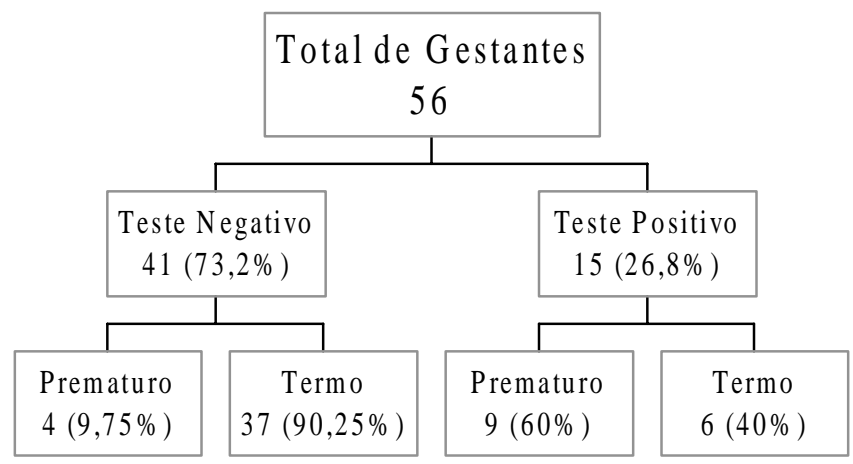

Figura 2 - Resultado da monitorização das contrações uterinas.

A idade gestacional média na qual o teste tornou-se positivo foi $30( \pm 2.9)$ semanas, sendo que o grupo com parto prematuro teve o teste positivo mais precocemente (29 \pm 3 semanas) do que o grupo com parto a termo (32 \pm 1 semanas). $\mathrm{O}$ intervalo médio entre teste positivo e o parto foi 7 ( \pm 3$)$ semanas. Tal intervalo foi significativamente menor entre os prematuros ( $6 \pm 3$ semanas), sendo que o intervalo mínimo foi de uma semana e o máximo de 10. Entre as gestantes com teste positivo e parto a termo, o intervalo até o parto foi de $7,2( \pm 4,4)$ semanas

A associação entre o teste positivo e o parto antes da $37^{\text {a }}$ semana revelou S, E, VPP e VPN de 69,23\% (9/13), 86,04\% (37/43), 60,0\% (9/15) e $90,24 \%(37 / 41)$ respectivamente.

O risco relativo, ou seja, a possibilidade de a gestante com teste positivo apresentar parto prétermo, foi 6,15 (variando de 2,22 a 17,03 ) vezes maior quando comparada com as que apresentaram teste negativo.

\section{Discussão}

A importância clínica do diagnóstico precoce do parto prematuro reside na possibilidade de aumento nos dias de gestação, mediante uma intervenção em tempo hábil. Tal intervenção permitiria uma melhora no peso fetal ao nascer, associada a uma diminuição da morbidade e mortalidade neonatal.

Por tais motivos, o objetivo primordial de qualquer programa de prevenção da prematuridade é a identificação acurada das pacientes com risco para o parto prematuro, tornando mais eficazes as medidas de prevenção.

Em virtude da etiologia multifatorial e das controvérsias quanto ao mecanismo fisiopatológico do parto prematuro, sua prevenção torna-se difícil. Porém, como o aumento das contrações uterinas é, na maioria das vezes, o desfecho desta cascata fisiopatológica, sua identificação pode ser utilizada como método de prevenção secundária.

Neste estudo, em que realizamos cerca de 11 horas de registros tocográficos por paciente, foi observado que as gestantes que evoluíram para parto prematuro apresentavam uma média de contrações horária significativamente maior, entre a $28^{\text {a }}$ e a $34^{\mathrm{a}}$ semana de gestação, do que as que evoluíram com parto de termo. Tais achados foram descritos por outros autores, sendo que seus resultados revelaram freqüência média inferior ao atual estudo ( 8 versus 4 a 5 contrações $/ h)^{11,15,16,18,21}$. Esta diferença se deve, provavelmente, ao fato de que neste estudo foram selecionadas pacientes com maior risco, sendo este representado pela elevada prevalência de antecedentes de parto prematuro (66\%) e pelo perfil sócio-econômico inferior à população dos outros trabalhos. Além disso, metade das pacientes $(50,2 \%)$ apresentavam nível de escolaridade restrito ao $1^{\circ}$ grau e na sua maioria incompleto. 
A prevalência de nascimento prematuro foi significativamente menor $(3 / 31-7 \%)$ entre as pacientes que apresentaram uma freqüência máxima inferior a 3 contrações/h em relação as que tiveram freqüência maior ou igual a 8 contrações/h (7/14 - 50\%). Nesta população a probabilidade do nascimento prematuro foi quatro vezes maior do que naquela. Estes resultados são semelhantes aos descritos por Main et al. ${ }^{16} \mathrm{e}$ sugerem que a presença de contrações uterinas é fator de risco para o parto prematuro, havendo uma relação direta entre contração e o nascimento prematuro.

Tais fatos justificam a utilização da monitorização das contrações uterinas como método preditivo do parto prematuro. Com este objetivo, utilizamos o ponto de corte de 4 contrações/h antes da $30^{\mathrm{a}}$ semana de gestação e 6 contrações/h após esta data para considerar o teste positivo, baseando-nos em dados da literatura mundial ${ }^{16,17,19}$.

Contudo, além da necessidade de valores preditivos elevados para assegurar ao obstetra um nível satisfatório de confiança, em face de resultados positivos ou negativos, é importante que o método possua bons índices de sensibilidade e de especificidade, o que possibilitaria a adoção de medidas preventivas. Neste estudo, a monitorização das contrações uterinas apresentou índices satisfatórios de sensibilidade $(69,2 \%)$ e especificidade $(86,0 \%)$ e um excelente VPN $(90,2 \%)$. Segundo Iams ${ }^{22,23}$, a S e o VPN elevados são pré-requisitos essenciais para testes de triagem, em que a falha do diagnóstico pode conduzir a graves conseqüências, sendo, neste estudo, o nascimento prematuro.

Bentley et al. ${ }^{17}$, considerando o teste positivo diante da presença de 4 contrações/h ou mais, persistentes em dois exames consecutivos, encontrou sensibilidade de 57\%, especificidade de $80 \%$ e valores preditivos positivo e negativo de $72 \%$ e 68\% respectivamente. Exceto pelo VPP, estes valores são inferiores aos nossos, provavelmente em virtude de o padrão-ouro utilizado ter sido o TPP, e não o nascimento pré-termo. Por outro lado, quando a população avaliada apresenta um baixo risco para o parto pré-termo, os resultados são insatisfatórios e os valores preditivos variam de 14 a 32\% ${ }^{16,22}$. Em tais situações, a associação com outros marcadores da prematuridade é apontada como solução ${ }^{13,22}$.

A capacidade de predição de nosso estudo revelou resultados semelhantes aos descritos por Morrison et al. ${ }^{20}$, cuja metodologia foi similar à nossa. Os relatos de Martin et al. ${ }^{24}$ foram os únicos que demonstraram uma sensibilidade $(85,7 \%)$ e um VPP (78\%) superiores aos nossos. Tal resultado se deve, provavelmente, ao critério utilizado para considerar o teste positivo, que foi a presença de 4 contrações/h ou mais em dois traçados tocográficos consecutivos, sendo orientada a hidratação oral e repouso no leito. Esta metodologia pode ter selecionado as gestantes com útero irritável e, desta forma, melhorado a confiabilidade do teste.

Todavia, em decorrência do baixo VPP (60\%) por nós encontrado, é necessário afastar a possibilidade de resultados falso-positivos diante de tocografias alteradas. Com este objetivo, poderse-ia utilizar artifício semelhante ao preconizado por Martin et al. ${ }^{24}$ ou associar outros marcadores clínicos ou bioquímicos do parto prematuro.

Ao confrontarmos os nossos resultados com os descritos por Bentley et al. ${ }^{17}$ e por Knuppel et al. ${ }^{25}$ observamos que nossa casuística apresentou um VPN maior, o que se justifica pelo fato de estes autores utilizarem o TPP como padrão-ouro e realizarem o teste apenas até a $34^{\mathrm{a}}$ semana de gestação, o que impossibilita a identificação de monitorizações alteradas após esta idade gestacional. Por outro lado, o melhor VPP (72\%) observado por estes autores pode ser justificado pela necessidade de haver duas tocografias consecutivas, com freqüência de 4 contrações $/ \mathrm{h}$ ou mais, para o teste ser considerado positivo. Este procedimento aumenta a acurácia do teste, possibilitando, desta maneira, a diminuição de testes falso-positivos.

Além dos valores preditivos satisfatórios, o intervalo entre o teste positivo e o parto foi de sete semanas, semelhante aos descritos na literatura ${ }^{15,16,18,20}$. Tal intervalo é suficiente para pesquisar o provável fator desencadeante, ou associar outros métodos de rastreamento, a fim de melhorar a acurácia da predição e, assim, intervir em tempo hábil, interceptando o fator patogênico, ou utilizando medidas profiláticas, tais como início precoce da tocólise ou emprego de corticoterapia, para minimizar os efeitos adversos da prematuridade.

Estes resultados demonstraram que a atividade uterina aumenta alguns dias antes do parto e que, as pacientes destinadas ao parto prematuro apresentam uma freqüência média horária significativamente maior que aquelas com parto a termo.

Baseado nestes resultados, o estudo das contrações uterinas por meio da monitorização externa deve ser utilizado em gestantes de risco para o parto prematuro espontâneo como primeira etapa da investigação. Os seus valores preditivos negativos satisfatórios tranqüilizam o obstetra diante de testes negativo e, desta forma, tornam desnecessário o emprego de medidas como 
internação hospitalar e tocólise. Por outro lado, quando o resultado for positivo, é mister o emprego simultâneo de outros métodos de predição, tal como a fibronectina fetal, o que possibilitaria a obtenção de melhores valores preditivos.

\section{SUMMARY}

Purpose: to evaluate the relationship between uterine contractions and premature delivery.

Methods: between February 1996 and July 1998, 73 high risk pregnant women for preterm delivery, between the 24th and 34 th weeks of gestation, were submitted to uterine contraction monitoring with tokodynamometers for 1 hour twice a week. The positive test was the presence of 4 contractions/h before the 30 th week of gestation, and after this time, 6 contractions $/ h$. Result: of 73 women, 17 patients (23.28\%) were excluded from the final analysis because they presented obstetric problems or unfavorable development for the final result. The rate of preterm delivery was $21.23 \%$ (13/56). The mean frequency of uterine contractions was greater in women with preterm delivery than in those with term delivery. The test presented sensitivity of $69.23 \%$, specificity of $86.04 \%$, positive predictive value of $60 \%$ and negative predictive value of $90.24 \%$.

Conclusion: negative tests are associated with a low risk of preterm birth. When the test is positive, association with other premature delivery markers is necessary to improve our ability to efficiently identify patients at risk for preterm delivery.

KEY WORDS: Uterine contractility. Prematurity. Labor.

\section{Referências}

1. Committee to Study the Prevention of Low Birth Weight, Institute of Medicine. Preventing low birth weight. Washington, DC: National Academy Press; 1985. p.175-251.

2. Morrison JC. Strategies in preventing preterm birth: evaluation of alternatives. J Maternal Fetal Invest $1991 ; 1: 67-72$.

3. March of Dimes Birth Defects Foundation (MDBDF). March of dimes statbook: statistic for Monitoring Maternal and Infant Health. [on line]. New York: MODBDF; 1997-1999. Available from: URL: http:/ /www.modimes.org/stats.htm

4. Perroni AG, Bittar RE, Fonseca ESVB, Messina ML, Marra KC, Zugaib M. Prematuridade eletiva: aspectos obstétricos e perinatais. Ginecol Obstet Bras 1999; 2:67-71.
5. Herron MA, Katz M, Creasy RK. Evaluation of a preterm birth prevention program: preliminary report. Obstet Gynecol 1982; 59: 452-6.

6. Papiernik E, Bouyer J, Collin D, Winisdoerffer G, Dreyfus J. Precocious cervical ripening and preterm labor. Obstet Gynecol 1986; 67:238-42.

7. Main DM, Gabbe SG, Richardson D, Strong S. Can preterm deliveries be prevented? Am J Obstet Gynecol 1985, 151: 892-8.

8. Owen J, Goldemberg RL, Davis RO, Kirk KA, Copper RL. Evaluation of risk scoring system as a predictor of preterm birth in a indigent population. Am J Obstet Gynecol 1990; 163: 87389.

9. Lockwood CJ, Senyei AE, Dische MR, Casal D, Shah $\mathrm{KD}$, Thung SN et al. Fetal fibronectin in cervical and vaginal secretions as a predictor of preterm delivery. N Engl J Med 1991; 325:669-74.

10.Bittar RE, Yamasaki AA, Sasaki S, Zugaib M. Cervical fetal fibronectin in patients at increased risk for preterm delivery. Am J Obstet Gynecol 1996; 175:178-81.

11.Kurtzman J, Goldsmith LJ, Gall S, Spinnato JA. Transvaginal versus transperineal ultrasound: a blinded comparison in the assessment of cervical length at mid-gestation. Am J Obstet Gynecol 1998; 179:852-7.

12.Yamasaki AA. Estudo do colo uterino através do toque vaginal e da ultra-sonografia transvaginal em gestantes de risco para o parto prematuro espontâneo. [Dissertação]. São Paulo: Faculdade de Medicina USP, 1997.

13.Rizzo G, Capponi A, Arduini MD, Lorido C, Romanini C. The value of fetal fibronectin in cervical and vaginal secretions and of ultrasonographic examination of the uterine cervix in predicting premature delivery for patients with preterm labor and intact membranes. Am J Obstet Gynecol 1996; 175:1146-51.

14.Yamasaki AA, Bittar RE, Fonseca ESB, Martinelli S, Sasaki S, Zugaib M. Prevenção do parto prematuro: emprego do toque vaginal e da ultrasonografia transvaginal. Rev Bras Ginecol Obstet 1998; 20:350-6.

15.Nageotte MP, Dorchester W, Porto M, Keegan KA, Freeman RK. Quantitation of uterine activity preceding preterm, term, and postterm labor. Am J Obstet Gynecol 1988; 158:1254-9.

16. Main DM, Katz M, Chiu G, Campion S, Gabbe SG. Intermittent weekly contraction monitoring to predict preterm labor in low risk women: a blinded study. Obstet Gynecol 1988; 72:757-61.

17.Bentley DL, Bentley JL, Watson DL, Welch RA, Martin RW, Gookin KS et al. Relationship of uterine contractility to preterm labor. Obstet Gynecol 1990; 76: 36S-38S. 
18.Katz M, Newman RB, Gill PJ. Assessment of uterine activity in ambulatory patients at high risk of preterm labor and delivery. Am J Obstet Gynecol 1986 ; 154:44-7.

19.Katz M, Gill PJ, Newman RB. Detection of preterm labor by ambulatory monitoring of uterine activity: a preliminary report. Am J Obstet Gynecol 1986; 68:773-8.

20.Morrison JC, Martin JN, Martin RW, Gookin KS, Wiser WL. Prevention of preterm birth by ambulatory assessment of uterine activity: a randomized study. Am J Obstet Gynecol 1987; 156:536-43.

21.Moore TR, Iams JD, Creasy RK, Burau KD, Davidson AL. Diurnal and gestacional patterns of uterine activity in normal human pregnancy. The uterine activity in pregnancy working group. Obstet Gynecol 1994; 83:517-23.
22.Iams JD. Prediction of preterm birth with ambulatory measurement of uterine contration frequency. Am J Obstet Gynecol 1998; 178: 2S.

23.Iams JD. Current status of home uterine activity monitoring. Clin Obstet Gynecol 1995; 38:771-9.

24.Martin RW, Gookin KS, Hill WC, Fleming AD, Knuppel RA, Lake MF et al. Uterine activity compared with symptomatology in the detection of preterm labor. Obstet Gynecol 1990; 76: 19S$23 \mathrm{~S}$.

25.Knuppel RA, Lake MF, Watson DL, Welch RA, Hill WC, Fleming $\mathrm{AD}$ et al. Preventing preterm birth in twin gestation: home uterine activity monitoring and perinatal nursing support. Obstet Gynecol 1990; 76:24S-27S. 